\section{Journal of Materials Research Editor's Report}

After a little over one year as Editor-inChief of the "official" journal of the Materials Research Society I would like to share a few thoughts about our journal. As Editor, I feel privileged to hold a position which lets me in effect eavesdrop on the most exciting developments in materials as they are happening. I can assure you that plenty of good science and engineering is happening in materials and that a good fraction of it is presented to the world through the Journal of Materials Research (JMR). From the earliest days of superconductivity, diamond thin films, and carbon tubes, IMR has been home to a substantial fraction of exciting papers. Characterization using electron microscopy, mechanical properties, microscopic and macroscopic defects are burgeoning areas with many $J M R$ papers. From the beginning, JMR has served as a home for exciting papers on ceramics ranging from sintering and phase equilibria to new electronic compositions. Electronic materials and metals are among the leaders in number and quality of $J M R$ papers.

Materials are already central to the solution of environmental problems and it is not an exaggeration to say that economic survival dictates that $a b$ initio green materials and processes must become more important. IMR has a special section in the March 1995 issue, edited by Julian Szekely of MIT, focused on Green Materials and Processes. We particularly encourage contributions on this subject on a continuing basis.

The need for laying the foundation for more scientifically based materials processing and its connection to competitiveness has been pointed out by numerous studies in the United States and other countries over the past decade. A special $J M R$ "focus issue" dedicated to materials processing, edited by Peter Esherick of Sandia National Laboratory, is planned for April 1996 with a manuscript deadline of August 1, 1995. A Call for Papers with further details [see page 61] will be printed shortly in JMR. We invite submissions for this issue and in the processing subject area on a continuing basis.

Rapid publication of brief manuscripts serves an important need in all scientific disciplines. The increased pace of materials science has caused me to revamp $J M R^{\prime}$ 's procedures for Communications with the recent appointment of Patrick K. Gallagher, of The Ohio State University, as Communications Editor. Prompt refereeing and rapid turnaround (30-day review) will now be SOP (see $J M R$ April 1995, Vol. 10, No. 4 for procedures). ceramics, composites, diamond;
A number of other areas of materials are growing and to ensure $J M R$ competence in these areas I have, during the past year, appointed a number of individual experts as New Principal Editors. These include

Robert Cammarata (Johns Hopkins University)-mechanical properties, especially microhardness;

Seshu Desu (Virginia Polytechnic Institute and State University)-processing, electronic materials;

Andrew Holmes (University of Cambridge, U.K.)-polymers, especially active polymers including conducting polymers, light-emitting polymers, NLO's, and photorefractivity;

Lisa Klein (Rutgers University)—sol-gel, electronic ceramics;

Zuhair Munir (University of California Davis) -combustion synthesis, phase equilibria;

Ian Robertson (University of Illinois at Urbana-Champaign) - microscopies and radiation effects in materials field microscopy;

Kenneth Sandhage (The Ohio State University) - thermodynamics, phase equilibria, superconductors, oxidation processing;

Yuh Shiohara (International Superconductivity Technology Center)-superconductivity;

Julian Szekely (Massachusetts Institute of Technology)-green materials and processes;

Carl Thompson (Massachusetts Institute of Technology)-silicon, electromigration, reliability, defects, thin-film microstructure, interconnect reliability; and

Yoshihisa Watanabe (National Defense Academy, Japan)-physical ceramics.

To better serve European and Asian countries we continue to have a special European Editor, Paul Siffert (CNRS, Laboratoire PHASE) and a Japanese Editor, Shigeyuki Sōmiya (The Nishi Tokyo University).

\section{Principal Editors in Europe include}

Rïdiger Bormann (GKSS, Research Center Geesthacht)-nanostructured materials, thermodynamics, metallic glasses, intermetallics; and

Jean-Paul Issi (Unité de Physico-Chimie et de Physique des Materiaux)-band structure, metals, superconductors.

\section{Principal Editors in Japan include}

Masao Doyama (The Nishi Tokyo University)-theory, computer modeling;

Shiushichi Kimura (Yamanashi University)-

Yoshiaki Kogure (The Nishi Tokyo
University)-theory, glasses;

Kiyoshi Kuribayashi (The Nishi Tokyo University) -ceramics, ferroelectrics, thin films; and

Shigehiko Yamada (The Nishi Tokyo University)-composites.

$J M R$ continues its traditional interest in radiation effect in materials and nuclear waste encapsulants, and continues to draw on a cadre of Principal Editors appointed in earlier years, including

R.P.H. Chang (Northwestern University)superconductivity, thin films;

Mildred S. Dresselhaus (Massachusetts Institute of Technology)-inorganics, carbon fibers and tubes, fullerenes;

Dean Face (DuPont Experimental Station)superconductors, piezoelectrics, thin films;

Frank Fradin (Argonne National Laboratory) - NMR, EPR, neutron diffraction, superconductivity, magnetism;

Harry Leamy (University of North Carolina at Charlotte)-thermodynamics, thin films, composites, crystal growth, characterization;

Werner Lutze (The University of New Mexico)-glasses, radioactive waste encapsulants;

Julia Phillips (AT\&T Bell Laboratories)superconductivity, thin films, epitaxy and ionbeam analysis;

Rustum Roy (The Pennsylvania State University)-high pressure, hydrothermal materials;

Karl Sieradzki (Arizona State University)mechanics and fracture, thin films/surfaces, scanning tunneling microscopy, corrosion/ electrochemistry, computer simulation of material behavior; and

David Turnbull (Harvard University)-theory, nucleation.

Lately, I have decided that reviews of leading edge fields in materials conveying a sense of excitement and introducing readers to hot areas at a rate of one per month would be a service to our readers and will begin these reviews in mid-1995. I solicit proposals by individuals willing to write such reviews. A title and brief abstract together with proposed date for submission should be sent to me.

Finally, my thanks to the authors and reviewers; the editorial staff (especially Helen Miller) at MRS headquarters in Pittsburgh, Pennsylvania; and especially to my fellow editors for their work, excellence, enthusiasm, and good humor.

ROBERT A. LAUDISE Editor-in-Chief Journal of Materials Research MIRIS 


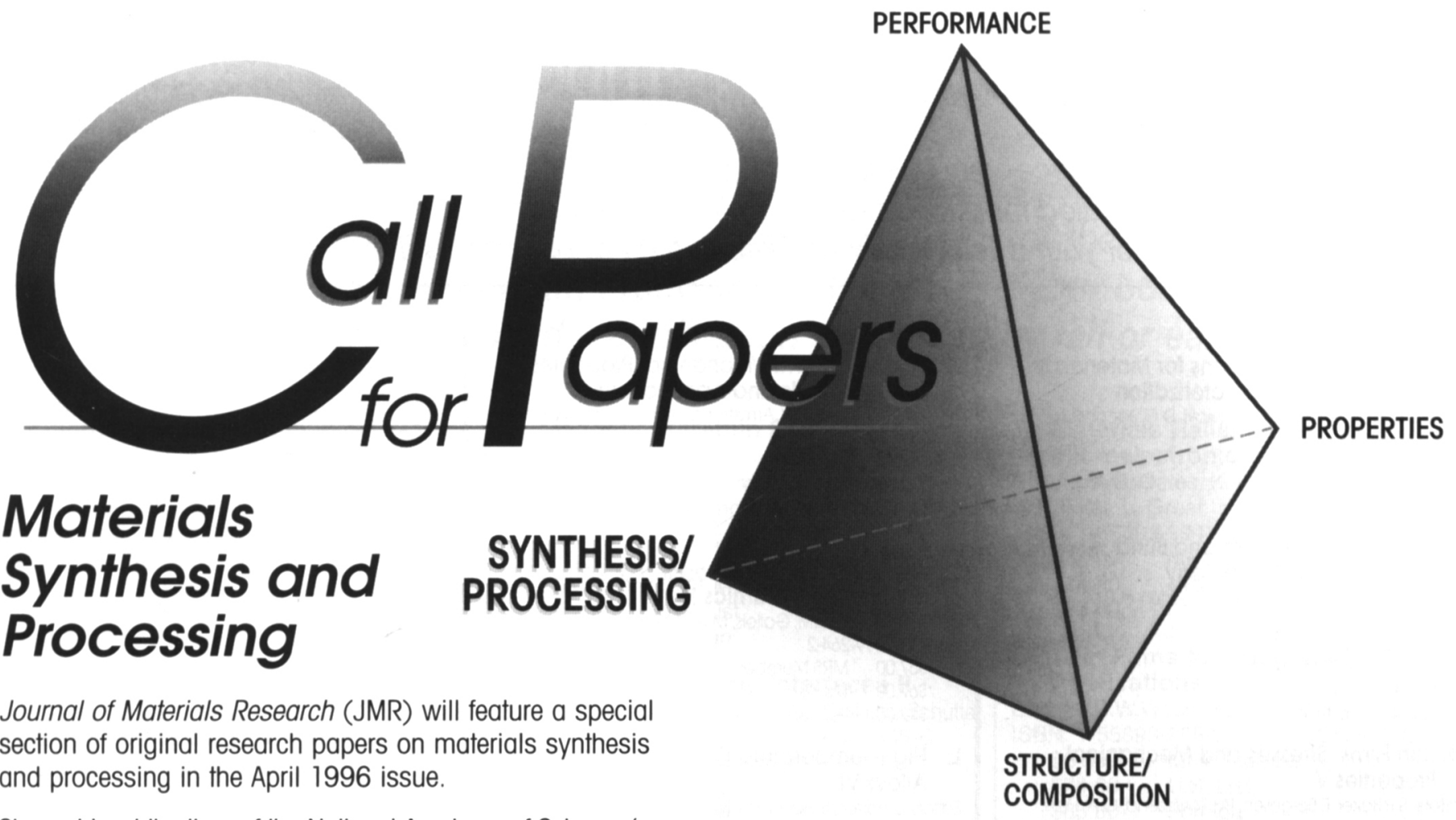

Since at least the time of the National Academy of Sciences' 1989 study "Materials Science and Engineering for the 90's" it has been generally recognized that in materials synthesis and processing there is "a serious weakness in the U.S. research effort" and "there are opportunities for progress in areas ranging from the basic science of synthesis and processing to materials manufacturing, that, if seized will markedly increase U.S. competitiveness."1

MRS has sponsored several symposia on processing issues, but while JMR has accepted papers it has not until now focused in that area.

The April 1996 issue will focus on synthesis and processing of engineered materials. For the purpose of this call for papers we will use the 1993 Federal Coordinating Council for Science, Engineering and Technology (FCCSET) definition of synthesis and processing as "the conversion of materials in their natural, atomic or molecular states to advanced materials suitable for specific intended uses, featuring precisely tailored properties and enhanced performance."2 Papers on all aspects of synthesis and processing as defined above are solicited. Atomic and molecular level engineering of materials processes, modeling of processes, fundamental understanding of generic processes such as plasma processing, chemical vapor deposition, sintering, etching, etc., are all suitable topics. Studies of yield and reliability of manufacturing processes as they are connected to processing of high-technology materials are appropriate. Papers on synthesis and processing of semiconductors, ceramics, composites and other "high-technology" materials would be of particular interest. Dr. Peter Esherick of Sandia National Laboratories will serve as editor for these papers.

To be considered for this issue, manuscripts must be received at the USA Editorial Office by August 1, 1995 No extensions of the deadline will be granted.

All manuscripts submitted for this special section of the April 1996 issue will be reviewed in a normal but expedited fashion. The top 15-20 manuscripts of all those accepted will be scheduled for publication in the April 1996 issue of JMR, appearing in the standard JMR format in a separate section of the issue. Any manuscripts that are accepted for publication but cannot be included in the group scheduled for publication in the April issue will be scheduled to appear in the next available issue of JMR.

Send your manuscript (one original plus three copies) for consideration to:

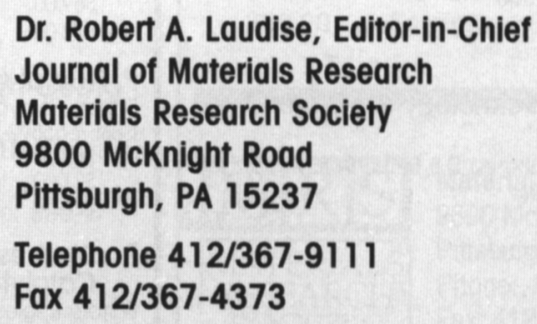

For information:

E-mail: miller@mrs.org MRS Homepage on World Wide Web: http://dns.mrs.org/

Indicate that the manuscripts are intended for the JMR April 1996 special section on Materials Synthesis and Processing.

1. 'Materials Science and Engineering for the 1990's - Maintaining Competitiveness in the Age of Materials," p. 3, National Academy Press, Washington, 1989.

2. "Advanced Materials and Processing: The Fiscal Year 1993 Program in Materials Science and Technology," p. 10, FCCSET Commiltee on Industry and Technology, NIST, Gaithersburg, MD, 1992. 
A: Beam-Solid Interactions for Materials Synthesis and Characterization

Editors: D.E. Luzzi, T.F. Heinz, M. Iwaki, D.C. Jacobson

ISBN: 1-55899-255-3

Code: 354-B

$\$ 67.00$ MRS Member

$\$ 75.00$ U.S. List

$\$ 80.00$ Foreign

B1: Evolution of Thin-Film and Surface

Structure and Morphology

Editors: B.G. Demczyk E.D. Williams, E. Garfunkel, B.M. Clemens,

J.E. CuOMO

ISBN: 1-55899-256-1

$\$ 69.00$ MRS Member

$\$ 77,00$ U.S. List

$\$ 82.00$ Foreign

B2: Thin Films: Stresses and Mechanical Properties V

Editors: S.P.Baker, P. Børgesen, P.H. Townsend, C.A. Ross,

C.A. Volkert

ISBN: 1-55899-257-X

$\$ 67.00$ MRS Member

$\$ 75.00$ U.S. List

$\$ 80.00$ Foreign

C: Structure and Properties of Interfaces in Ceramics

Editors: D.A. Bonnell, U. Chowdhry, M. Rühle

ISBN: 1-55899-258-8

$\$ 57.00$ MRS Member

$\$ 67.00$ U.S. List

$\$ 72.00$ Foreign

F: Microcrystalline and Nanocrystalline Semiconductors

Editors: L. Brus. M. Hirose, R.W. Colltins, F. Koch. C.C. Tsal

ISBN: 1-55899-259-6

$\$ 72.00$ MRS Member

$\$ 80.00$ U.S. List

$\$ 85.00$ Foreign

G: Science and Technology of Fullerene Materials

Editors: P. Bernier, T.W. Ebbesen, D.S. Bethune, R.M. Metzger.

Y. Chiang J.W. Mintmire

ISBN: 1-55899-260-X

$\$ 62.00$ MRS Member

Code: 359-B

$\$ 70.00$ U.S. List

$\$ 75,00$ Foreign

\section{II: Materials for Smart Systems}

Editors: S. Troller-McKinstry, K. Uchino, M. Wun-Fogle,

E.P.George, S. Takahashi

ISBN: 1-55899-261-8

$\$ 57.00$ MRS Member

Code: $360-\mathrm{B}$

$\$ 67.00$ U.S. List

$\$ 72.00$ Foreign

12: Ferroelectric Thin Films IV

Editors: S.B. Desu, B.A. Tuttle, R. Ramesh, T. Shiosaki

ISBN: 1-55899-262-6

$\$ 59.00$ MRS Member

$\$ 69.00$ U.S. List

$\$ 74.00$ Foreign
$\mathrm{Jb}$ :Grain-Size and Mechanical PropertiesFundamentals and Applications

Editors: N.J. Grant, R.W. Armstrong, M.A. Otooni, T.N. Baker. K. Ishizaki

ISBN: 1-55899-263-4

$\$ 59,00 \quad$ MRS Member

Code: $362-B$

$\$ 69.00$ U.S. List

$\$ 74.00$ Foreign

K: Chemical Vapor Deposition of Refractory Metals and Ceramics III

Editors: W.Y. Lee, B.M. Gallois, M.A. Pickering

ISBN: 1-55899-264-2

$\$ 57.00$ MRS Member

$\$ 67,00$ U.S. List

$\$ 72.00$ Foreign

L: High-Temperature Ordered Intermetallic Alloys VI

Editors: J. Horton, S. Hanada, I. Baker, R.D. Noebe, D. Schwartz

ISBN: 1-55899-265-0

$\$ 72.00 \quad$ MRS Member

$\$ 80.00$ U.S. List

$\$ 85.00$ Foreign

M: Ceramic Matrix Composites-Advanced High-Temperature Structural Materials

Editors: R.A. Lowden, J.R. Hellmann, M.K. Ferber, S.G. DiPietro, K.K. Chawla

ISBN: 1-55899-266-9

$\$ 57,00$ MRS Member

code: $365-\mathrm{B}$

$\$ 67.00$ U.S. List

$\$ 72.00$ Foreign

$\mathrm{N}$ : Dynamics in Small Confining Systems II

Editors: J.M. Drake, S.M. Troian, J. Klaffer, R. Kopelman

ISBN: 1-55899-267-7

Code: $366-B$

$\$ 59,00$ MRS Member

$\$ 69.00$ U.S. List

$\$ 74.00$ Foreign

\section{P: Fractal Aspects of Materials}

Editors: Fereydoon Family, B. Sapoval, P. Meakin, R. Wool

ISBN: 1-55899-268-5

$\$ 62.00$. MRS Member

$\$ 71.00$ U.S. List

$\$ 76.00$ Foreign

T: Synthesis and Properties of Advanced Catalytic Materials

Editors: E. Iglesia, P. Lednor, D. Nagaki, L. Thompson

ISBN: 1-55899-270-7

$\$ 57.00$ MRS Member

$\$ 67.00$ U.S. List

$\$ 72.00$ Foreign

U: Solid State Ionics IV

Editors: G-A. Nazri, J-M. Tarascon, M. Schreiber

ISBN: 1-55899-271-5

$\$ 67.00$ MRS Member

$\$ 77.00$ U.S. List

$\$ 82.00$ Foreign

$\mathrm{Va} / \mathrm{Vb}$ : Microstructure of Cement-Based Systems / Bonding and Interfaces in Cementitious Maferials

Editors: S. Diamond, F.P. Glasser, L.D. Wakeley / S. Mindess,

J. Skalny, L. Roberts

ISBN: 1-55899-272-3

$\$ 57.00$ MRS Member

$\$ 67.00$ U.S. List

$\$ 72.00$ Foreign
$W_{1}$ : Advances in Porous Materials

Editors: S. Komarneni, D.M. Smith, J.S. Beck

ISBN: 1 1-55899-273-1

$\$ 57.00$ MRS Member

$\$ 67.00$ U.S. List

$\$ 72.00$ Foreign

W2: Hollow and Solid Spheres and Microspheres-Science and Technology

Associated With Their Fabrication and

Application

Editors: M. Berg. T. Bernat, D. L. Wilcox, Sr., J.K. Cochran, Jt. D. Kellerman

ISBN: 1-55899-274-X

$\$ 62.00$ MRS Member

$\$ 71.00$ U.S. List

$\$ 76.00$ Foreign

Y: Microstructure of Irradiated Materials

Editors: I.M. Robertson, S.J. Zinkle, L.E. Rehn, W.J. Phythign

ISBN: 1-55899-275-8

$\$ 62.00$ MRS Member

$\$ 71.00$ U.S. List

$\$ 76.00$ Foreign

Zb: Materials for Optical Limiting

Editors: R. Crane, K. Lewis, E. V Stryland, M. Khoshnevisan

ISBN: 1-55899-276-6

$\$ 62.00$ MRS Member

$\$ 71.00$ U.S. List

$\$ 76.00$ Foreign

AA: Applications of Synchrotron Radiation Techniques to Materials Science II

Editors: D. L. Perry, N. Shinn, K. D'Amico, G. ice, L. Terminello

ISBN: 1-55899-277-4

$\$ 59.00$ MRS Member

$\$ 69.00$ U.S. List

$\$ 74.00$ Foreign

BB: Neutron Scattering in Materials Science II

Editors: D.A. Neumann, T.P. Russell, B.J. Wuensch

ISBN: 1-55899-278-2

$\$ 67.00$ MRS Membe

$\$ 77.00$ U.S. List

$\$ 82.00$ Foreign

Code: $375-8$

MRS Materials Research Society

LIS 9800 Mcknight Road

MATERIALS Pittsburgh, PA 15237

RESEARCH Phone: 412-367-3012

SOCIETY Fax: 412-367-4373

In Europe, Africa and the Middle East:

Clarke Associates-Europe, Ltd.

Fourth Floor, The Rackhay

Queen Charlotte Street

Bristol BS1 4HJ

ENGLAND

Phone: 0272268864

Fax: 0272226437

Free shipping on all prepaid orders, If not prepaid

standard shipping \& handling charges of $\$ 3$ per book will

be added to each order.

Contact MRS for special shipping. 


\section{The Newest Interdisciplinary Research on Advanced Materials 1995 Spring Meeting Symposium Proceedings

A: Amorphous Silicon Technology-1995 Editors: M. Hack, E.A. Schiff, M. Powell, A. Matsuda, A. Madan

ISBN: $1-55899-280-4$

$\$ 66.00 \quad$ MRS Member

$\$ 75.00$ U.S. List

$\$ 85.00 \quad$ Foreign

B: Defect- and Impurity-Engineered Semiconductors and Devices

Editors: S. Ashok, J. Chevallier, I. Akasaki,

N.M. Johnson, B.L. Sopori

ISBN: $1-55899-281-2 \quad$ Code: $378-B$

$\$ 72.00 \quad$ MRS Member

$\$ 83.00$ U.S. List

$\$ 95.00$ Foreign

C: Strained Layer Epitaxy-Materials, Processing, and Device Applications Editors: J. Bean, E. Fitzgerald, J. Hoyt, K-Y. Cheng ISBN: 1-55899-282-0 Code: $379-B$
$\$ 62.00$
MRS Member
$\$ 71.00$ U.S. List

$\$ 81.00$ Foreign

D: Materials-Fabrication and Patterning at the Nanoscale

Editors: C.R.K. Marrian, K. Kash, F. Cerrina,

M. Lagally

ISBN: $1-55899-283-9 \quad$ Code: $380-B$

$\$ 72.00$ MRS Member

$\$ 83.00$ U.S. List

$\$ 95.00$ Foreign

F: Low-Dielectric Constant MaterialsSynthesis and Applications in Microelectronics

Editors: T-M. Lu, S.P. Murarka, T.S. Kuan, C.H. Ting

ISBN: 1-55899-284-7 Code: 381-B

$\$ 72.00 \quad$ MRS Member

$\$ 83.00$ U.S. List

$\$ 95.00 \quad$ Foreign

G: Structure and Properties of Multilayered Thin Films

Editors: T.D. Nguyen, B.M. Lairson, B.M. Clemens, K. Sato, S-C. Shin

ISBN: 1-55899-285-5 Code: 382-B

$\$ 74.00 \quad$ MRS Member

$\$ 85.00 \quad$ U.S. List

$\$ 98.00$ Foreign

I: Mechanical Behavior of Diamond and Other Forms of Carbon

Editors: M.D. Drory, M.S. Donley, D. Bogy, J.E. Field

ISBN: 1-55899-286-3

Code: $383-B$

$\begin{array}{ll}\$ 67.00 & \text { MRS Member } \\ \$ 77.00 & \text { U.S. List }\end{array}$

$\$ 89.00 \quad$ Foreign
L: Magnetic Ultrathin Films, Multilayers and Surfaces

Editors: A. Fert, H. Fujimori, G. Guntherodt,

B. Heinrich, W.F. Egelhoff, Jr., E.E. Marinero,

R.L. White

ISBN: 1-55899-287-1 Code: 384-B

$\$ 68.00 \quad$ MRS Member

$\$ 78.00 \quad$ U.S. List

$\$ 90.00 \quad$ Foreign

N: Polymer/Inorganic Interfaces II

Editors: L. Drzal, N.A. Peppas, R.L. Opila, C. Schutte

ISBN: $1-55899-288-X \quad$ Code: $385-B$
$\$ 64.00$
MRS Member
$\$ 74.00$ U.S. List
$\$ 85.00 \quad$ Foreign

O: Ultraclean Semiconductor

Processing Technology and Surface Chemical Cleaning and Passivation

Editors: M. Liehr, M. Hirose, M. Heyns, H. Parks

ISBN: 1-55899-289-8 Code: 386-B

$\begin{array}{ll}\$ 57.00 & \text { MRS Member } \\ \$ 63.00 & \text { U.S. List } \\ \$ 72.00 & \text { Foreign }\end{array}$

P: Rapid Thermal and Integrated Processing IV

Editors: J.C. Sturm, J.C. Gelpey, S.R.J. Brueck, A. Kermani, J.L. Regolini

ISBN: 1-55899-290-1 Code: 387-B

$\$ 57.00 \quad$ MRS Member

$\$ 63.00$ U.S. List

$\$ 72.00$ Foreign

Q: Film Synthesis and Growth Using Energetic Beams

Editors: H.A. Atwater, J.T. Dickinson, D.H. Lowndes, A. Polman

ISBN: 1-55899-291-X Code: 388-B

$\$ 68.00 \quad$ MRS Member

$\$ 78.00 \quad$ U.S. List

$\$ 89.00 \quad$ Foreign

R: Modeling and Simulation of Thin-Film Processing

Editors: C.A. Volkert, R.J. Kee, D.J. Srolovitz,

M.J. Fluss

ISBN: $1-55899-292-8 \quad$ Code: $389-B$

$\$ 66.00 \quad$ MRS Member

$\$ 77.00 \quad$ U.S. List

$\$ 88.00 \quad$ Foreign

S: Electronic Packaging Materials Science VIII

Editors: R.C. Sundahl, K.A. Jackson, K-N. Tu, P. Børgesen

ISBN: 1-55899-293-6 Code: 390-B

$\$ 63.00$ MRS Member

$\$ 72.00$ U.S. List

$\$ 83.00$ Foreign
T: Materials Reliability in Microelectronics $\mathbf{V}$

Editors: A.S. Oates, K. Gadepally, R. Rosenberg, W.F. Filter, L. Greer

ISBN: 1-55899-294-4

$\$ 64.00 \quad$ MRS Member

$\$ 74.00$ U.S. List

$\$ 85.00 \quad$ Foreign

U: Thin Films for Integrated Optics Applications

Editors: B.W.Wessels, D.M. Walba

ISBN: 1-55899-295-2 Code: 392-B

$\$ 64.00$ MRS Member

$\$ 74.00$ U.S. List

$\$ 85.00 \quad$ Foreign

W: Materials for Electrochemical Energy Storage and ConversionBatteries, Capacitors and Fuel Cells

Editors: D.H. Doughty, B. Vyas, J.R. Huff,

T. Takamura

ISBN: 1-55899-296-0 Code: 393-B

$\$ 64.00 \quad$ MRS Member

$\$ 74.00 \quad$ U.S. List

$\$ 85.00 \quad$ Foreign

Z: Polymers in Medicine and Pharmacy Editors: A.G. Mikos, K.W. Leong, M.L. Radomsky, J.A. Tamada, M.J. Yaszemski

ISBN: 1-55899-297-9

$\$ 68.00 \quad$ MRS Member

$\$ 78.00$ U.S. List

$\$ 89.00$ Foreign

Code: 394-B

\section{(⿻)}

Materials Research Society 9800 McKnight Road Pittsburgh, PA 15237 Phone: 412-367-3012 Fax: $412-367-4373$

In Europe, Africa and the Middle East:

Clarke Associates-Europe, Ltd.

Fourth Floor, The Rackhay

Queen Charlotte Street

Bristol BS1 4HJ

ENGLAND

Phone: 0272268864

Fax: 0272226437

Free shipping on all prepaid orders. If not prepaid, standard shipping \& handling charges of $\$ 3$ per book will be added to each order.

Contact MRS for special shipping. 


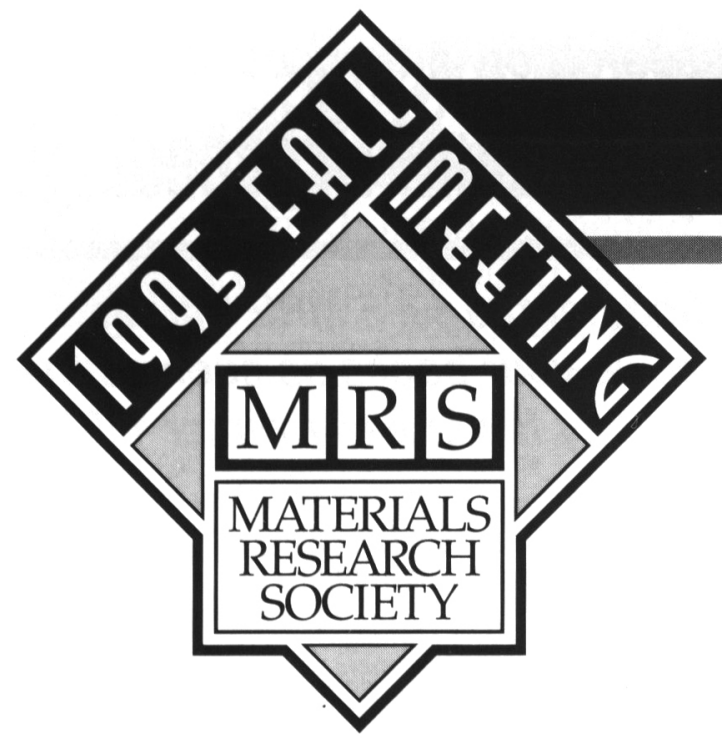

November 27 - December I, 1995

Boston Marriott Hotel and Westin Hotel/Copley Place

Sheraton Boston Hotel

Boston, Massachusetts

\section{SYMPOSIA}

AAA: Gallium Nitride and Related Materials

Russell D. Dupuis, The University of Texas at Austin, Phone (512) 471-0537, Fax (512) 471-8575, E-mail: dupuis@ece.utexas.edu; Fernando A. Ponce, Xerox PARC, Phone (415) 812-4199, Fax (415) 812-4140, E-mail: ponce@parc.xerox.com; John A. Edmond Cree Research Inc. Phone (919) 361-5709. Fax (919) 361-4630, E-mail: 74660.3702 @compuserve.com; Shuji Nakamura, Nichia Chemical Industries, Japan, Phone (81) 884-22-2311, Fax (81) 884-23-1802, E-mail: shuj@ @ichia.co.jp

A: Ion-Solid Interactions for Materials Modification and Processing Daryush lla, Alabama A\&M University, Phone (205) 851-5866, Fax (205) 851-5868, E-mail: aamdxi01@asnaam.aamu.edu; Lloyd R. Harriott. AT\&T Bell Laboratories, Phone (908) 582-4922, Fax (908) 582-3901, Email: Irh@physics.att.com; David B. Poker Oak Ridge National Laboratory, Phone (615) 576-8827, Fax (615) 576-6720, E-mail: dbp@ornl.gov: Yang-Tse Cheng. General Motors Corporation, Phone (810) 986-0939, Fax (810) 986-8697, E-mail: cheng@gmr.com; Thomas W. Sigmon, Arizona State University, Phone (602) 965-5580, Fax (same as phone), E-mail: sigmon@enuxsa.eas.asu.edu

B: Advanced Laser Processing of Materials - Fundamentals and Applications Rajiv Singh, University of Florida, Phone (904) 392-1032, Fax (904) 392-3771; David Norton, Oak Ridge National Laboratory, Phone (615) 574-5965, Fax (615) 5744814; J. Narayan, North Carolina State University, Phone (919) 515-7874, Fax (919) 5157642; Jeff Cheung, Rockwell International Science Center, Phone (805) 373-4144, Fax (805) 373-4775; L.D. Laude, University of Mons Hainaut Belgium, Fax (32) 65-373427

C: Thermodynamics and Kinetics of Phase Transformations James S. Im, Columbia University, Phone (212) 854-8341, Fax (212) 854-3054; A. Lindsay Greer, Cambridge University, United Kingdom, Phone (44) 223-334308 Fax (44) 223-334373; Byungw0o Park, Georgia Institute of Technology, Phone (404) 894-2544, Fax (404) 853-9140; G. Brian Stephenson, IBM T.J. Watson Research Center, Phone (914) 945-3008, Fax (914) 945-2141

\section{D: Evolution of Epitaxial Structure and Morphology}

Andrew Zangwill, Georgia Institute of Technology, Phone (404) 894-7333, Fax (404) 8539958, E-mail: zangwill@zang1.physics.gatech.edu; David Jesson, Oak Ridge National Laboratory, Phone (615) 574-6281, Fax (615) 574-4143, E-mail: ejp@solid.ssd.ornl.gov; David Chambliss, IBM-Almaden Research Center, Phone (408) 927-2243, Fax (408) 927-2100, E-mail: chamb@almaden.ibm.com; Roy Clarke, University of Michigan, Phone (313) 764-4466, Fax (313) 764-2193, E-mail: royc@umich.edu

E: Metastable Metal-Based Phases and Microstructures Giorgio Mazzone, ENEA, Italy, Phone (39) 6-30484354, Fax (39) 6-30484729, E-mail: mazzone g@casaccia.enea.it: Robert D. Shull, National Institute of Standards and Technology, Phone (301) 975-6035, Fax (301) 975-2128, E-mail: shull@enh.nist.gov; Robert S. Averback, University of Illinois, Urbana-Champaign, Phone (217) 333-4302, Fax (217) 333-2736, E-mail: raverbac@uy.ncsa.uiuc.edu; Ruediger Bormann, GKSS Forschungszentrum, Germany, Phone (49) 41-52872562, Fax (49) 41-52872534 E-mail: ruediger.bormann@gkss.de; Ronald F. Ziolo, Xerox Corp., Phone (716) 422-3341, Fax (716) 422-1035, E-mail: ziolo.wbst128@xerox.com

\section{C/MT}

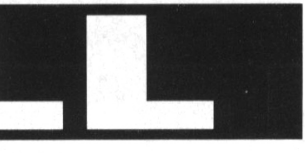

MATERIALS RESEARCH SOCIETY

800 McKnight Road, Pittsburgh, PA 15237-6006

(4I2) 367-3004, Fax (4I2) 367-4373

Mail: info@mrs.org

F: Defects in High-Temperature Superconductors - Characterization and Relations to Processing and Properties

Kenneth C. Goretta, Argonne National Laboratory, Phone (708) 252-7761, Fax (708) 252-3604 Susan E. Babcock University of Wisconsin. Madison, Phone (608) 263-5696, Fax (608) 263-1087; James N. Eckstein, Varian Associates, Phone (415) 424-5081 Fax (415) 424-6988; Kohji Kishio, University of Tokyo, Japan, Phone (81) 3-3812-2111 Fax (81) 3-5689-0574; Eva M. Olsson, Chalmers University of Technology, Sweden, Phone (46) 31-7723316, Fax (46) 31-7723224

G: Epitaxial Oxide Thin Films II

D.K. Fork, Xerox Palo Alto Research Center, Phone (415) 812-4121, Fax (415) 812-4140, E-mail: fork.parc@xerox.com; R.M. Wolf, Philips Research, The Netherlands, Phone (31) 40743748, Fax (31) 40-743365, E-mail: wolfr@prl.philips.nl; J.S. Speck, University of California, Santa Barbara Phone (805) 893-8005. Fax (805) 893-8486. E-mail: speck@surface.ucsb.edu T. Shiosaki, Kyoto University, Japan, Phone (81) 75-753-5309, Fax (81) 75-753-5749, E-mail: tshiosak@kuee.kyoto-u.ac.jp

H: Silicide Thin Films - Fabrication, Properties, and Applications Ray Tung, AT\&T Bell Laboratories, Phone (908) 582-6895, Fax (908) 582-4228, E-mail: H@clockwise attcom; Karen Maex, IMEC , Belgium, Phone (32) 16-281-358, Fax (32) 16-281-214, E-mail: maex@imec.be; Paul W. Pellegrini, Rome Laboratory, Hanscom AFB, Phone (617) 377-3699, Fax (617) 377-5041, E-mail: pellegrini@eastlonex.rl.af.mil; Leslie H. Allen, University of Illinois, Urbana, Phone (217) 333-7918, Fax (217) 244-

1631, E-mail: allen@ux1.cso.uiuc.edu

I: $\quad$ Polycrystalline Thin Films II - Structure, Texture, Properties and Applications Harold J. Frost, Dartmouth College, Phone (603) 646-3444, Fax (603) 646-3856, E-mail: harold.frost@dartmouth.edu; Michael A. Parker, IBM Storage Systems Division, Phone (408) 256-4039, Fax (408) 256-5151, E-mail: mparker@sjevm13.vnet.ibm.com Caroline A. Ross, Komag Inc. Phone (408) 957-4400, Fax (408) 263-9449, E-mail: caross@leland.stanford.edu; Elizabeth A. Holm, Sandia National Laboratories, Phone (505) 844-7669, Fax (505) 844-1543, E-mail: eaholm@sandia.gov

J: In Situ Electron and Tunneling Microscopy of Dynamic Processes Renu Sharma, Arizona State University, Phone (602) 965-4541, Fax (602) 965-9004, E-mail: sharma@csss.la.asu.edu; Marija Gajdardziska-Josifovska, University of Wisconsin Milwaukee, Phone (414) 229-4965, Fax (414) 229-5589, E-mail: mgj@alpha2.csd.uwm.edu; Pratibha L. Gai, DuPont Science and Engineering Laboratories, Phone (302) 695-9203, Fax (302) 695-1664, E-mail: gaip@@esvax.dnet.dupont.com: Robert Sinclair, Stanford University, Phone (415) 723-1102, Fax (415) 725-4034; Lloyd J. Whitman, Naval Research Laboratory, Phone (202) 404-8845, Fax (202) 767-3321, E-mail: Iwhitman@stm2.nrl.navy.mil

K: $\quad$ Surface/Interface and Stress Effects in Electronic Material Nanostructures S.M. Prokes, Naval Research Laboratory, Phone (202) 767-2799, Fax (202) 767-4290; R.C. Cammarata, The Johns Hopkins University, Phone (410) 516-5462, Fax (410) 516-5293 K.L. Wang, University of California, Los Angeles, Phone (310) 825-1609, Fax (310) 2068495; A. Christou, University of Maryland, Phone (301) 405-5208, Fax (301) 314-2029

L: Diagnostic Techniques for Semiconductor Materials Processing Orest J. Glembocki, Naval Research Laboratory, Phone (202) 767-2799, Fax (202) 767-4290, E-mail: glembocki@estd.nrl.navy.mil; Stella W. Pang, University of Michigan, Phone (313) 936-2962, Fax (313) 763-9324, E-mail: pang@eecs.umich.edu; Fred H. Pollak, Brooklyn College, Phone (718) 951-5356, Fax (718) 951-4871, E-mail: fhpbc@cunyvm.cuny.edu; Francis Celii, Texas Instruments Inc., Phone (214) 995-7764, Fax (214) 995-7785, E-mail: celii@resbld.csc.ti.com; Clivia M. Sotomayor Torres, University of Glasgow, United Kingdom, E-mail: c.sotomayor-torres@elec.gla.ac.uk

M: Disordered Materials and Interfaces - Fractals, Structure, and Dynamics Herman Z. Cummins, City College of New York (CUNY), Phone (212) 650-6921, Fax (212) 650-6923,E-mail: cummins@sci.ccny.cuny.edu; H. Eugene Stanley, Boston University, Phone (617) 353-2617. Fax (617) 353-3783, E-mail: hes@buphyk.bu.edu; Douglas J. Durian, University of California, Los Angeles, Phone (310) 206-2645, Fax (310) 825-5734, E-mail: durian@physics.ucla.edu; David L. Johnson, Schlümberger-Doll Research Center, Phone (203) 431-5227, Fax (203) 438-3819, E-mail: djohnson@ridgefield.sdr.slb.com

\section{N: Complex Fluids}

Dave Pine, Exxon Research and Engineering Co., Phone (908) 730-2968, Fax (908) 730 3232, E-mail: djpine@erenj.com; Fred MacKintosh, University of Michigan, Phone (313) 763-9891, Fax (313) 764-6843, E-mail: fcm@umich.edu; Bob Meyer, Brandeis University, Phone (617) 736-2870, Fax (617) 736-2915, E-mail: meyer@binah.cc.brandeis.edu 


\section{FOR PAPPERS}

\section{0: Self-Assembling Materials and Structures}

Jeffrey M. Calvert, Naval Research Laboratory, Phone (202) 404-6008, Fax (202) 767-9594, E-mail: jcalvert@cbmse.nrl.navy.mil; Thomas Mallouk, Pennsylvania State University, Phone (814) 863-9637, Fax (814) 863-8403, E-mail: tom@chem.psu.edu; Paul Yager, University of Washington, Phone (206) 543-6126. Fax (206) 543-6124, E-mail: yager@bioeng.washington.edu; Edwin A. Chandross, AT\&T Bell Laboratories, Phone (908) 582-3542, Fax (908) 582-3609, eac@clockwise.att.com

P: Materials Theory, Simulations, and Parallel Algorithms Etthimios Kaxiras, Harvard University, Phone (617) 495-7977, Fax (617) 495-0416 E-mail: kaxiras@cmt.harvard.edu; John Joannopoulos, Massachusetts Institute of Technology, Phone (617) 253-4806, Fax (617) 253-2562, E-mail: joannop@mit.edu Priya Vashishta, Louisiana State University, Phone (504) 388-1157 or (504) 388-1342, Fax (504) 388-5855, E-mail: priyav@bit.csc.Isu.edu; Rajiv K. Kalia, Louisiana State University, Phone (504) 388-1112, Fax (504) 388-5855, E-mail: kalia@bit.csc.Isu.edu

Q: Fracture - Instability Dynamics, Scaling, and Ductile/Brittle Behavior Jack Mecholsky, University of Florida, Phone (904) 392-9692, Fax (904) 846-0326, E-mail: imech@mse.ufl.edu; Robin Blumberg Selinger, National Institute of Standards and Technology Phone (301) 975-2716. Fax (301) 926-8349. E-mail: selinger@enh.nist.gov; Anders Carlsson, Washington University, Phone (314) 935-5739, Fax (314) 935-6219, E-mail: aec@howdy.wustl.edu; Edwin R. Fuller, Jr., National Institute of Standards and Technology, Phone (301) 975-5795, Fax (301) 990-8729, E-mail: fuller@tiber.nist.gov

R: Mechanisms of Chemical Degradation of Cement-Based Systems Karen L. Scrivener, Imperial College, United Kingdom, Phone (44) 71-5946723, Fax (44) 71-5843194, E-mail: kls@ic.ac.uk; Waldemar A. Klemm, Construction Technology Laboratories, Inc., Phone (708) 965-7500, Ext. 541, Fax (708) 965-6541; J. Francis Young, University of Illinois, Urbana, Phone (217) 244-6210, Fax (217) 244-6917 E-mail: young@ux1.cso.uiuc.edu; Vagn Johansen, G.M. Idorn, Denmark, Phone (45) 45-986730 Fax (45) 45-986932: Gregory J. McCarthy, North Dakota State University, Phone (701) 231-7193, Fax (701) 231-8831, E-mail: gmccarth@vml.nodak.edu

S: Covalent Ceramics III - Science and Technology of Non-Oxides

Alain E. Kaloyeros, University at Albany-SUNY, Phone (518) 442-4533, Fax (518) 442-

4585, E-mail: ak127@rachel.albany.edu; Prashant N. Kumta, Carnegie Mellon University, Phone (412) 268-8739, Fax (412) 268-7596, E-mail: pk12@andrew.cmu.edu; Gary S. Fischman : Alfred University, Phone (607) 871-2284, Fax (607) 871-2317, E-mail: fischman@xray.alfred.edu; Contact: Aloysius F. Hepp, NASA Lewis Research Center, Phone (216) 433-3835, Fax (216) 433-6106, E-mail: sehepp@mars.lerc.nasa.gov; John J. Sullivan, MKS Instruments, Phone (508) 975-2350, Fax (508) 975-0093

\section{T: Electrically Based Microstructural Characterization}

Rosario A. Gerhardt, Georgia Institute of Technology, Phone (404) 894-6886, Fax (404) 853-9140, E-mail: rosario.gerhardt@mse.gatech.edu; S. Ray Taylor, University of Virginia, Phone (804) 982-5788, Fax (804) 982-5799, E-mail: sit6p@uva.pcmail. virginia.edu; Edward J. Garboczi, National Institute of Standards and Technology, Phone (301) 975-6708, Fax (301) 990-6891, E-mail: garbocz@enh.nist.gov

\section{$\mathrm{U}: \quad$ Materials Inspired by Biology}

Bob Birge, Syracuse University, Phone (315) 443-1900, Fax (315) 443-4070, E-mail: bob@birge.cat.syr.edu; Joe Cappello, Protein Polymer Technologies, Phone (619) 5586064, Fax (619) 558-6477: R. Malcolm Brown, Jr., University of Texas, Austin, Phone (512) 471-3364, Fax (512) 471-3573; Janos Fendler, Syracuse University, Phone (315) 443-4146, Fax (315) 443-4869, E-mail: cmes@suvm

V: Scientific Basis for Nuclear Waste Management XIX

William M. Murphy, Southwest Research Institute, Phone (210) 522-5263, Fax (210) 522-5155, E-mail: wmurphy@swri.edu; Dieter A. Knecht, Lockheed Idaho Technologies Company, Phone (208) 526-3627, Fax (208) 526-3499, E-mail: dieter@pmafire.inel.gov

W: Electrical, Optical, and Magnetic Properties of Organic Solid State Materials Alex K. - Y. Jen, ROITechnology, Phone (908) 422-3709, Fax (908) 422-3783; Mike F. Rubner, Massachusetts Institute of Technology, Phone (617) 253-4477, Fax (617) 258-7874; Gary E. Wnek, Rensselaer Polytechnic Institute, Phone (518) 276-2885, Fax (518) 276-4887; Larry R. Dalton, University of Southern California, Phone (213) 740-8768, Fax (213) 740-2701. Charles Y-C. Lee Air Force Office of Scientific Research, Phone (202) 767-4963, Fax (202) 404-7475; Long Y. Chiang, National Taiwan University, Taiwan, Phone (886) 2-362-5507, Fax (886) 2-365-5404

$\mathrm{X}$ : $\quad$ Frontiers of Materials Research

Michael J. Aziz, Harvard University, Phone (617) 495-9884, Fax (617) 495-9837 E-mail: aziz@das.harvard.edu; Berend T. Jonker, Naval Research Laboratory, Phone (202) 404-8015, Fax (202) 767-1697, E-mail: jonker@anvil.nrl.navy.mil; Leslie J. Struble, University of Illinois-Urbana, Phone (217) 333-2544, Fax (217) 333-9464, E-mail: Istruble @cern.ce.uiuc.edu

Y: $\quad$ Long-Term Performance Issues in Polymers - Chemistry and Physics Sindee L. Simon, University of Pittsburgh, Phone (412) 624-9635, Fax (412) 624-9639, E-mail: ssimon@engrng.pitt.edu; Greg B. McKenna, NIST, Phone (301) 975-6752, Fax (301) 869-3239, E-mail: greg@micf.nist.gov; Harvey E. Bair, AT\&T Bell Laboratories, Phone (908) 582-2381, Fax (908) 582-5570

Z: Thin Films and Surfaces for Bioactivity and Biomedical Applications Cathy Cotell, Naval Research Laboratory, Phone (202) 767-4433, Fax (202) 767-5301, E-mail: cotell@nrl3.nrl.navy.mil; George Grobe Bausch \& Lomb, Phone (716) 338-8228, Fax (716) 338-5304; Steven M. Gorbatkin, Oak Ridge National Laboratory, Phone (615) 576-6502, Fax (615) 576-8135, E-mail: gss@ornl.gov; Anne E. Meyer, SÜNY-Buffalo, Phone (716) 829-3560, Fax (716) 835-4872
AA: Low-Cost Manufacturing of Materials

Joel P. Clark, Massachusetts Institute of Technology, Phone (617) 253-6885, Fax (617) 258-8836, E-mail: jpclark@mit.edu; Sudhir S. Savkar, General Electric Company Phone (518) 387-5808, Fax (518) 387-5752 E-mail: savkar@crd ge.com. John H. Hopps, National Science Foundation, Phone (703) 306-1811, Fax (703) 306-0515, E-mail: jhopps@nsf.gov; Alan I. Taub, Ford Research Laboratory, Phone (313) 248-4039, Fax (313) 390-0514. E-mail: ataub@smail.srl.ford.com; Lee Hong $\mathrm{Ng}$, Hewlett-Packard, Phone (415), 857-5726, Fax (415) 857-4646

BB: Metal-Organic Chemical Vapor Deposition of Electronic Ceramics II Seshu B. Desu, Virginia Tech, Phone (703) 231-6820, Fax (703) 231-8919, E-mail: desu@vtvm1.cc.vt.edu; David B. Beach, Oak Ridge National Laboratory, Phone (615) 574 5024, Fax (615) 574-4939, E-mail: beachdb@ornl.gov: Peter C. Van Buskirk. Advanced Technology Materials Inc., Phone (203) 794-1100, Ext. 130, Fax (203) 830-4116, E-mail: pvanb@atmi.com

CC: Spectroscopy of Heterojunctions

Norman Tolk, Vanderbilt University, Phone (615) 322-2786, Fax (615) 343-7263 Giorgio Margaritondo, Ecolé Polytech Federale, Switzerland, Phone (41) 21-6934471, Fax (41) 21-6934666: Enrique Viturro, Xerox Webster Research Center, Phone (716) 422-3753, Fax (716) 422-1035

DD: Diamond for Electronic Applications

Pehr Pehrsson, Naval Research Laboratory, Phone (202) 767-3579, Fax (202) 767 3321, E-mail: pehrsson@nrlfs1.nrl.navy.mil; Trevor Humphreys. Research Triangle Institute, Phone (919) 541-6888, Fax (919) 541-6515, E-mail: trevor@es.rti.org Charles Beetz, Fairfield University, Phone (203) 355-4662, Fax (203) 254-4277, E-mail: cbeetz@fair1.fairfield.edu; Alan Collins, King's College London, United Kingdom, Phone (44) 171-873-2584, Fax (44) 171-873-2160, E-mail: atc@ipg.ph.kcl.ac.uk Kumar Das, Tuskegee University, Phone (205) 727-8994, Fax (205) 727-8090, E-mail: dask@acd.tusk.edu; David Dreifus, Kobe Steel USA, Inc., Phone (919) 549-9823, Fax (919) 549-8460, E-mail: 74224.1056@compuserve.com

EE: Optoelectronic Materials - Ordering, Composition Modulation, and SelfAssembled Structures

Eric D. Jones, Sandia National Laboratories, Phone (505) 844-8752. Fax (505) 844 8985, E-mail: edjones@sandia.gov; Pierre Petroff, University of California, Santa Barbara, Phone (805) 893-8511, Fax (805) 893-8502, E-mail: petroff@engrhub.ucsb.edu; Angelo Mascarenhas, National Renewable Energy Laboratory, Phone (303) 384-6608, Fax (303) 384-6481, E-mail: amascar@nrel.nrel.gov; Raj Bhat, Bell Communications Research, Phone (908) 758-3385, Fax (908) 758-4372, E-mail: bhat2@nyquist.bellcore.com

FF: Fullerenes, Buckytubes, and Related Materials - Science and Applications Vinayak P. Dravid, Northwestern University, Phone (708) 467-1363, Fax (708) 491-7820 E-mail·v-dravid@nwu edu; Joseph Shinar lowa State University, Phone (515) 294-8706, Fax (515) 294-0689, shinar@ameslab.gov; Jack E. Fischer, University of Pennsylvania, Phone (215) 898-6924, Fax (215) 573-2128, E-mail: fischer@sol1. Irsm.upenn.edu; Robert C. Haddon, AT\&T Bell Laboratories, Phone (908) 582-3200, Fax (908) 582-3958, E-mail: haddon@allwise.att.com

GG: Decomposition, Combustion, and Detonation Chemistry of Energetic Materials Thomas B. Brill, University of Delaware, Phone (302) 831-6079, Fax (302) 831-6335, E-mail: brill@brahms.udel.edu; Thomas P. Russell, Naval Research Laboratory, Phone (202) 767-9030, Fax (202) 404-8119, E-mail: russell3@chem.nrl.navy.mil: William C. Tao Lawrence Livermore National Laboratory, Phone (510) 423-0499, Fax (510) 422-2382, E-mail: ta01@|lnl.gov; Robert B. Wardle, Thiokol Corporation Phone (801) 863-6156, Fax (801) 863-2271, E-mail: bwardle@aol.com

\section{MEETING CHAIRS}

Michael J. Aziz, Division of Applied Sciences, Harvard University, 29 0xford Street, Cambridge, MA 02138, Phone (617) 495-9884 Fax (617) 495-9837, aziz@das.harvard.edu

Berend T. Jonker, Code 6345, Naval Research Laboratory, 4555 Overlook Avenue, SW, Washington, DC 20375-5343, Phone (202) 404-8015, Fax (202) 787-1697, E-mail: jonker@anvil.nrl.navy.mil

Leslie J. Struble, 2129 Newmark Civil Engineering Lab, University of Illinois-Urbana, 205 North Mathews Avenue, Urbana, IL 61801 2352, Phone (217) 333-2544, Fax (217) 333-9464, E-mail: Istruble @cern.ce.uiuc.edu 


\section{Ouer I650 Leading Illaterıals Scientists at Your fingertips The
The Advanced Engineering Materials Research Profile Directory} focuses on the university, national laboratory and federally funded laboratory communities. In addition to address, telephone and e-mail information, each profile identifies the researcher's focus area from over 30 topical areas, and provides insight into the laboratory procedures and techniques used.

A comprehensive description of research projects underway puts you in touch with the right resources and contacts. The database is easily searched by any of the over 30 fields, including research description, geographical area and more. And all data is provided directly by the researchers profiled, so that information is current and accurate.

\section{The Advanced Engineering Materials Research Profile Directory is} available in print directory or computer database format ... or in a convenient and money-saving packaged set. ORDER TODAY, and use it to:
$\nabla$ locate new technologies
$\nabla$ recruit high-level expertise
$\boldsymbol{\nabla}$ identify early stage research
$\checkmark$ find respected expert witnesses
$\nabla$ highlight collaborative research
$\checkmark$ target lab equipment decision-makers
$\boldsymbol{\nabla}$ consult with leading authorities

\section{Computer Database \\ \$395 Order Code: AEMRI-B (IBM format) \\ AEMRM-B (Macintosh format)}

(specify tab-delimited or comma-delimited text; available in 3.5" diskette only)

Print Directory

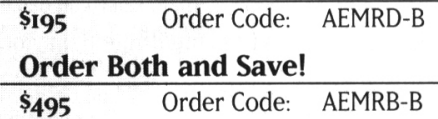

\section{Databasefeatures:}

Subject: North American Materials Research

Number of Records: 1667

Database Fields Per Record: $4 \mathrm{I}$

Database Electronic Field Size: Approximately 1426K (ASCII text)

Directory Pages: 289

Indexed By: Contact Name, Research Expertise

Ordec from:

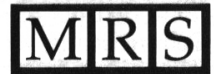

MATERIAIS

RESEARCH

SOCIETY
MATERIALS RESEARCH SOCIETY

Publications Department 9800 McKnight Road Pittsburgh, PA $15237-6006$ U.S.A Phone: 412-367-3012; Fax: 412-367-4373

The Advanced Engineering Materials Research Profile Directory is a joint project of the Materials Research Society and Synergistic Technologies, Inc.

\section{NOW AVAILABLE FROM MRS}

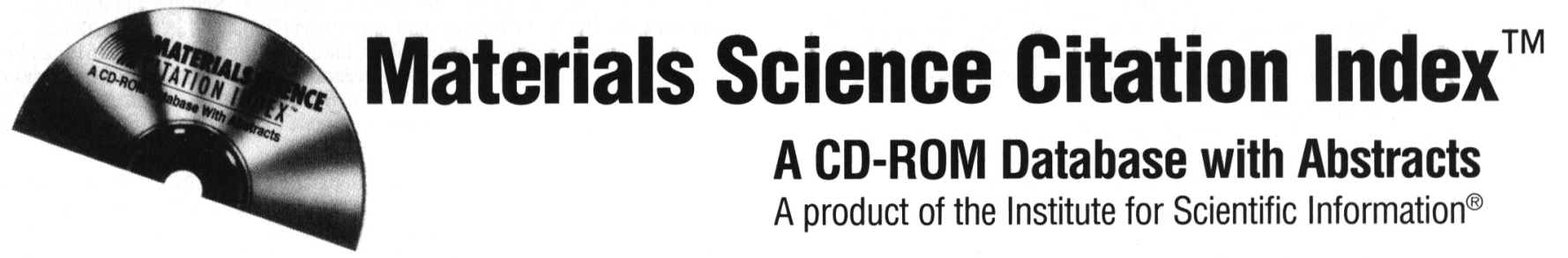

Materials Science Citation Index (MSCI) provides researchers with extensive bibliographic information -including author, title, source publication, author-supplied abstract and cited references-from over 1300 books, journals and conference proceedings related to materials science. Total coverage exceeds 90,000 articles per year. Published bimonthly on compact disc, the Materials Science Citation Index covers all areas of materials science, including ceramics, semiconductors, superconductors, metals and metallurgy, thin films, plastics and polymer engineering, composites, biomaterials, adhesives, minerals, fabrics and fibers...plus electronics, surface science, applied physics, methods of extraction, processing and manufacturing, and more.

Unique features of the $\mathbf{M S C I}$ include: searchable author abstracts; cited reference searching, which enables you to take a known paper and find more recent papers that cite it; and Related Records ${ }^{\mathrm{TM}}$, which extends the power of citation indexing by linking and displaying the articles that have one or more references in common.

A 1995 subscription includes back-year data to 1991 , for over 350,000 source items!

For more information contact:
Code: MSCI-B

$\$ 799.00$ MRS Members U.S. $\$ 819.00$ MRS Members Foreign

Free Sample Trial Available
\$994.00 Non-Members U.S. $\$ 1014.00$ Non-Members Foreign

\section{M|RTS MATERIALS RESEARCH SOCIETY}

MATERIALS RESEARCH SOCIETY
Publications Department • 9800 McKnight Road • Pittsburgh, PA 15237-6006

Phone: 412-367-3012 • FAX: 412-367-4373 